\title{
Revisão por Pares na Aprendizagem de Modelagem de Sistemas: concepção de uma ferramenta de suporte
}

\author{
Romualdo Azevedo, Patricia Chourio, Alberto Castro, Bruno Gadelha \\ ${ }^{1}$ Instituto de Computação - Universidade Federal do Amazonas (UFAM) \\ 69.080 - 900 - Manaus - AM - Brasil \\ \{romualdo.costa, ppcp, alberto, bruno\}@icomp.ufam.edu.br
}

\begin{abstract}
The adoption of collaborative learning techniques for software engineering education provides the opportunity for discussing software modeling issues considering multiple points of view. As a consequence, students are expected to develop better models for software problems. To do so, it is necessary to develop or adapt tools that support teachers and students when adopting this approach. This paper describes a study on the use of the Collaborative Learning Peer Review technique in a System Modeling courses. As a result, we have a set of requirements for a tool to support this technique for teaching modeling software with UML (Unified Modeling Language).
\end{abstract}

Resumo. A aprendizagem colaborativa para o ensino de modelagem de sistemas oportuniza a discussão sobre a resolução de problemas baseados em múltiplos pontos de vista. Como consequência espera-se que os estudantes desenvolvam melhores modelos para tratar problemas de software. Para tanto é necessário desenvolver ou adaptar ferramentas que ajudem professores e alunos durante a adoção de tal abordagem. Este artigo descreve um estudo sobre o uso da técnica de aprendizagem colaborativa Revisão por Pares em aulas de modelagem de software. Como resultado, apresenta-se um conjunto de requisitos para a construção de uma ferramenta que dê suporte a técnica de Revisão por Pares no ensino de modelagem de sistemas com UML (Unified Modeling Language).

\section{Introdução}

Modelagem de sistemas é uma tarefa não trivial onde alunos de Engenharia de Software enfrentam muitas dificuldades durante o processo de aprendizagem. No meio acadêmico é comum que alunos aprendam a modelar sistemas utilizando diagramas da Unified Modeling Language (UML) por serem muito utilizados na indústria. No entanto, é frequente que os estudantes demonstrem dificuldades em compreender a sintaxe e a semântica de tais diagramas [Ma 2017]. Neste contexto, a interação entre pessoas possibilita que os membros do grupo identifiquem inconsistências e falhas em seu raciocínio ao se depararem com outros pontos de vistas e entendimentos [Quarto et al. 2017].

Nesse cenário, o uso de técnicas de aprendizagem colaborativa pode diminuir as dificuldades durante o aprendizado de modelagem com UML, pois desenvolvem diversas habilidades de crítica e autocrítica. Uma das técnicas de aprendizagem colaborativas é a Revisão por Pares [Jenal et al. 2012] na qual os alunos realizam uma atividade e revisam as atividades dos colegas, levando a melhores respostas às atividades solicitadas. $\mathrm{O}$ uso 
VIII Congresso Brasileiro de Informática na Educação (CBIE 2019)

Anais do XXX Simpósio Brasileiro de Informática na Educação (SBIE 2019)

da Revisão por Pares no ensino de modelagem de sistemas pode proporcionar diferentes visões sobre o problema, resultando em modelos de software melhores e melhoria nas habilidades dos alunos em lidar com diferentes pontos de vista [Barkley et al. 2014].

Assim, este artigo apresenta um estudo que visa verificar como a técnica de Revisão por Pares pode auxiliar no processo de aprendizagem de modelagem de sistemas utilizando UML. Para tanto, a técnica foi aplicada em duas turmas do curso de Engenharia de Software da Universidade Federal do Amazonas, com alunos do primeiro e quinto períodos, com e sem o apoio de ferramenta de suporte. Como resultado, este artigo discute a viabilidade sobre o uso da técnica em disciplinas de modelagem de software e apresenta um conjunto de requisitos, bem como um protótipo de ferramenta de Revisão por Pares para modelos de software.

Este artigo está organizado da seguinte forma: a Seção 2 apresenta os trabalhos relacionados; na Seção 3 é apresentada a metodologia utilizada na pesquisa; a Seção 4 apresenta a pesquisa exploratória realizada; na Seção 5 é descrito o processo de elicitação de requisitos utilizado; a Seção 6 apresenta os resultados da pesquisa que consistem em um conjunto de requisitos e o protótipo de uma ferramenta para a aplicação da Revisão por Pares em turmas de modelagem de software; e na Seção 7, as conclusões são apresentadas.

\section{Trabalhos Relacionados}

Em [Bruning 2016], o autor relata que desenvolvedores precisam trabalhar em conjunto para criar uma solução que atenda as necessidades do cliente. Desta forma, é descrito o desenvolvimento de uma ferramenta de suporte a modelagem colaborativa para os diagramas de classes da UML. Como resultado, são apresentados testes funcionais e validação da ferramenta por possíveis usuários.

[Neto et al. 2004] apresentam uma análise de requisitos da Revisão por Pares na aprendizagem buscando identificar e documentar as principais características necessárias para construir um software para aprendizagem colaborativa. Os requisitos foram elicitados utilizando os diagramas de casos de uso UML e por meio da aplicação de um processo de desenvolvimento de software. A principal contribuição deste trabalho está no levantamento, documentação e apresentação dos requisitos de software para apoiar a Revisão por Pares na aprendizagem.

Em [Junior et al. 2016] é relatada a construção de um sistema de suporte para Revisão por Pares. Para elicitar os requisitos do sistema o autor utilizou técnicas de entrevista, prototipação e análise de sistemas correlatos. Como resultado foram apresentados os requisitos do sistema, o feedback dos testes realizados e o resultado final do sistema que permitia a Revisão por Pares.

Diferente dos trabalhos apresentados, este relato apresenta um estudo sobre a viabilidade de uso da Revisão por Pares na aprendizagem de modelagem de sistemas com UML e propõe um conjunto de requisitos para uma ferramenta de suporte. O trabalho de [Bruning 2016] aborda uma ferramenta de modelagem colaborativa, porém, sem viés educacional. Esta pesquisa vai ao encontro dos trabalhos de [Neto et al. 2004] e [Junior et al. 2016], que apresentam abordagens complementares similares a esta. 
VIII Congresso Brasileiro de Informática na Educação (CBIE 2019)

Anais do XXX Simpósio Brasileiro de Informática na Educação (SBIE 2019)

\section{Metodologia}

A pesquisa iniciou-se por uma revisão da literatura com o objetivo de verificar como técnicas de aprendizagem colaborativa podem ser incorporadas ao ensino de Engenharia de Software, mais especificamente no que diz respeito à modelagem de sistemas. Assim, verificou-se a possibilidade de investigar a adoção da Revisão por Pares nesse contexto. Para tanto, fez-se necessário observar sua aplicabilidade em sala de aula. Dessa forma, realizou-se uma pesquisa exploratória que se dividiu em: (a) análise em sala de aula e (b) análise utilizando o ambiente Moodle como intermediador. A partir dos resultados da pesquisa exploratória, realizou-se um levantamento de requisitos para uma ferramenta de suporte por meio das técnicas de: (i) Brainstorm; (ii) Entrevista e, (iii) Prototipação, que resultou-se em um protótipo para uma ferramenta de aplicação da Revisão por Pares em aulas de modelagem de sistemas.

Pesquisa Exploratória: realizada com o objetivo de verificar a adequação da Revisão por Pares na aprendizagem de modelagem de sistemas, consistiu em duas etapas: (a) análise em sala de aula: referente à aplicação da revisão por pares em sala de aula, sem auxílio tecnológico, e; (b) análise utilizando o Moodle: referente à aplicação da Revisão por Pares utilizando o recurso "Laboratório de Avaliação" disponibilizado na plataforma Moodle.

Brainstorm: foi realizado para coletar as ideias que os participantes da pesquisa exploratória tiveram sobre os requisitos para uma ferramenta de apoio à aplicação da técnica. Os participantes falavam em voz alta o que acreditavam ser requisitos e, ao fim da listagem, cada ponto foi discutido verificando sua viabilidade e necessidade na ferramenta.

Entrevista: a entrevista ocorreu em forma de focus group para coletar a opinião dos alunos sobre a técnica de Revisão por Pares e o funcionamento do recurso "Laboratório de Avaliação" do Moodle. Os alunos listavam as vantagens e desvantagens sobre a técnica e eram estimulados a comentar sobre elas enquanto eram filmados (com seus consentimentos) pelo moderador.

Prototipação: foi realizada para validar os requisitos elicitados e verificar como os requisitos seriam implementados na ferramenta.

\section{Pesquisa Exploratória}

A pesquisa exploratória foi realizada em turmas de duas disciplinas do curso de Engenharia de Software na Universidade Federal do Amazonas, a saber: Fundamentos de Engenharia de Software (FES), ofertada a alunos do primeiro período, onde contou-se com a participação de 49 alunos, e; Engenharia de Requisitos e Análise de Sistemas (ERAS), que consiste em uma disciplina do quinto período e contou com a participação de 11 alunos. As duas disciplinas eram ministradas pelo mesmo professor.

Assim, para a condução da pesquisa, o professor explicou seus objetivos, bem como a dinâmica da técnica de Revisão por Pares. Em seguida, foi disponibilizado um Termo de Consentimento Livre e Esclarecido que foi assinado por todos os participantes. Durante a aplicação da técnica, foram utilizados diagramas de casos de uso (UC) e diagramas de classes (DC) por representarem as visões mais comuns da UML como a visão funcional (diagrama de casos de uso) e a visão estrutural (diagrama de classes), além de 
VIII Congresso Brasileiro de Informática na Educação (CBIE 2019)

Anais do XXX Simpósio Brasileiro de Informática na Educação (SBIE 2019)

serem os mais utilizados na indústria de software [Silva et al. 2018].

No total, houve três aplicações da técnica Revisão por Pares nesta pesquisa exploratória. A primeira com a participação das duas turmas, FES e ERAS, ocorreu em sala de aula e utilizou-se o diagrama de Casos de Uso. Na primeira aplicação, utilizou-se um checklist como Peer Review Information de modo a auxiliá-los durante a correção dos diagramas, já que os alunos não tinham experiência no desenvolvimento de Casos de Uso. A segunda aplicação ocorreu com auxílio de mediação tecnológica através do recurso "Laboratório de Avaliação" disponível no Moodle.

Esta segunda aplicação ocorreu somente com a turma de ERAS. Para a terceira aplicação da técnica foi utilizado o diagrama de Classes. Esta técnica foi aplicada somente em sala de aula e somente na turma de ERAS. A turma de FES não participou desta rodada pois a ementa da disciplina não cobria tal assunto. Neste terceiro momento não foi utilizado checklist. A Tabela 1 sumariza todas essas informações:

Tabela 1. Revisão por Pares nas turmas de Engenharia de Software

\begin{tabular}{|c|c|c|}
\hline Item & $\begin{array}{c}\text { Fundamentos de Engenharia } \\
\text { de Software }\end{array}$ & $\begin{array}{c}\text { Engenharia de Requisitos } \\
\text { e Análise de Sistemas }\end{array}$ \\
\hline Quantidade de Alunos & 49 & 11 \\
\hline Diagrama Utilizado & UC & UC e DC \\
\hline Revisão por Pares & UC com checklist & $\begin{array}{c}\text { UC com e sem checklist e } \\
\text { DC sem checklist }\end{array}$ \\
\hline Elicitação & Brainstorm & Entrevista \\
\hline Mediação Tecnológica & Não & Sim (Moodle) \\
\hline
\end{tabular}

A subseção a seguir detalha como ocorreu a condução das aplicações da Revisão por Pares em sala de aula.

\subsection{Análise em Sala de Aula}

A análise em ambiente real ou análise em sala de aula foi realizada em duas turmas. Um dos pesquisadores explicou como funcionava a técnica, descrevendo-a e tirando dúvidas sobre seu funcionamento. Os alunos dispuseram de 15 minutos para elaborar o diagrama e 10 minutos para realizar a revisão no diagrama dos colegas. Então, os alunos dispuseram de mais 15 minutos para realizar as correções sugeridas. Apesar do nível dos alunos serem diferentes o enunciado utilizado para a pesquisa foi o mesmo nas duas turmas. Tratava-se da elaboração de um diagrama de casos de uso de acordo com uma pequena descrição de um cenário. Como na turma de FES os alunos não tinham conhecimento sobre diagramas da UML, os pesquisadores decidiram que eles participassem de apenas uma das rodadas de Revisão por Pares. Os alunos da turma de ERAS participaram da Revisão por Pares em casos de uso em duas rodadas, na primeira utilizando um checklist como peer review information e na segunda sem o checklist pois tinham conhecimento prévio necessário por já estarem no quinto período e familiarizados com o diagrama.

Os passos de aplicação da técnica foram os mesmos para os dois diagramas. No entanto, o tempo para resolução foi maior para o diagrama de classes pois o professor da disciplina o considerava mais trabalhoso. Após as rodadas de Revisão por Pares utilizando os diagramas de casos de uso, foi disponibilizado aos alunos um questionário 
VIII Congresso Brasileiro de Informática na Educação (CBIE 2019)

Anais do XXX Simpósio Brasileiro de Informática na Educação (SBIE 2019)

online para a avaliação da qualidade da técnica de aprendizagem colaborativa de Revisão por Pares. Durante o questionário os alunos responderam sobre as suas experiências ao utilizar a técnica de aprendizagem colaborativa e suas percepções sobre a aprendizagem. Os participantes avaliaram vinte itens sobre a aplicação da técnica de Revisão por Pares. O grau de concordância para mensuração dos dados utilizado foi o da escala de Likert, sendo 1 para Discordo Totalmente e 5 para Concordo Totalmente. No questionário, as primeiras perguntas, de 1 a 8, diziam respeito à percepção da aprendizagem, já as perguntas seguintes, de 9 a 13, eram sobre aspectos colaborativos, as perguntas finais, de 14 a 20, eram referentes à facilidade e utilidade de uso da técnica. Os resultados são apresentados na Figura 1:

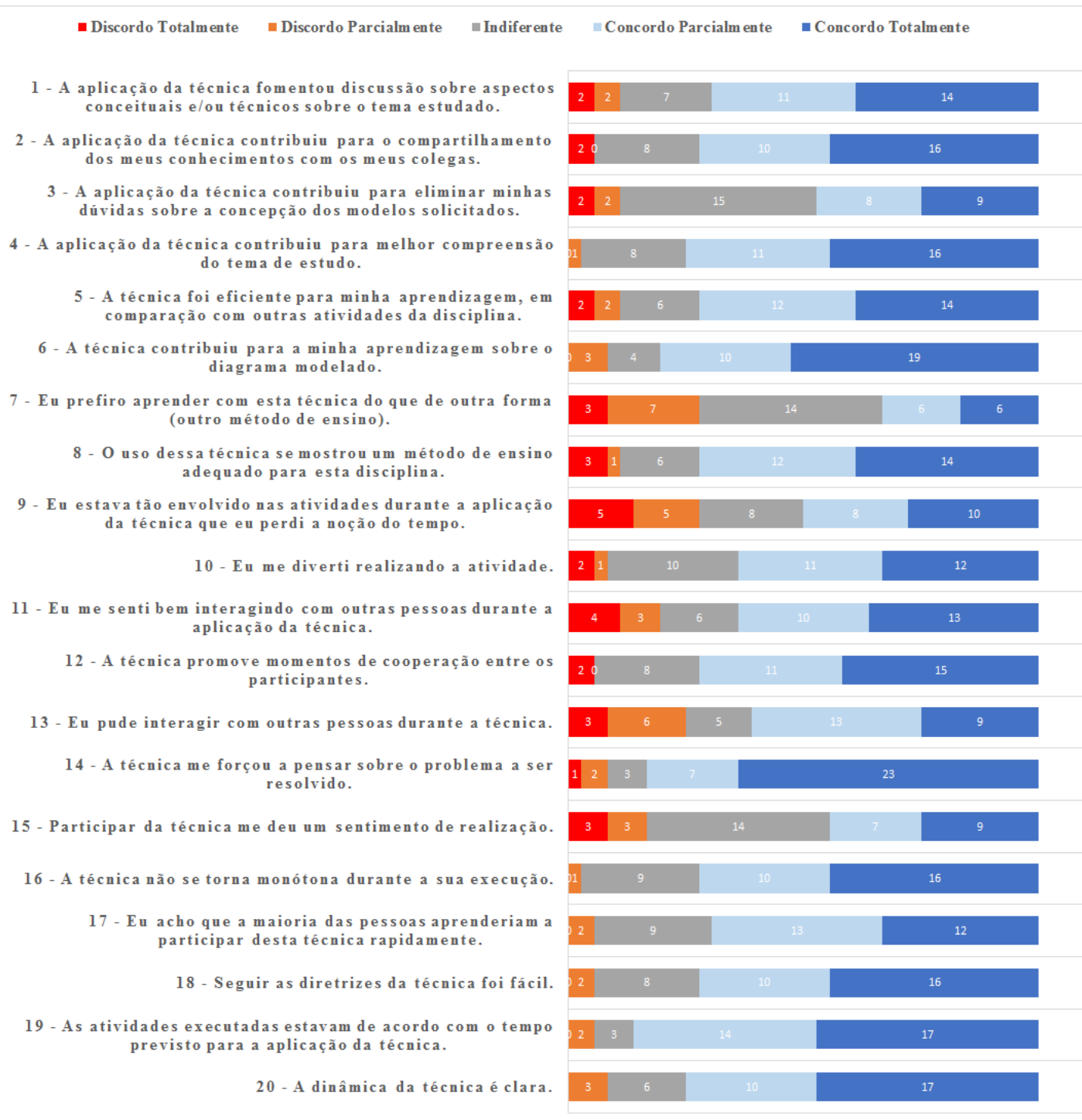

Figura 1. Análise das respostas do questionário

Diante do gráfico apresentado na Figura 1 é possível perceber que a técnica forçou os alunos a pensar sobre o problema a ser resolvido e que contribuiu para a aprendizagem sobre os diagramas modelados. No entanto, poucos alunos responderam que a técnica eli- 
VIII Congresso Brasileiro de Informática na Educação (CBIE 2019)

Anais do XXX Simpósio Brasileiro de Informática na Educação (SBIE 2019)

minou suas dúvidas sobre a concepção dos modelos. Isto se dá porque a técnica realmente não ensina os modelos, a técnica visa a colaboração dos alunos perante o aprendizado. A maioria dos alunos relatou que preferiria aprender com outra forma de ensino ao invés de utilizar a Revisão por Pares. Neste caso, pensa-se que os alunos ainda estão acomodados diante dos métodos tradicionais de ensino, onde somente o professor fala e o aluno ouve, e quando se deparam com uma técnica de colaboração como esta eles têm que se desvencilhar de sua zona de conforto. Entretanto, a maioria dos alunos relatou que seguir as diretrizes da técnica foi fácil, se divertiram durante a utilização da técnica e o uso da técnica mostrou-se adequado para a utilização nesta disciplina. Conclui-se que a Revisão por Pares é um método adequado para ser utilizado durante o aprendizado de modelagem de sistemas já que possibilita aos alunos uma nova forma de desenvolver soluções e aprendam a fazer críticas e autocríticas.

\subsection{Análise Utilizando o Moodle}

Esta etapa da pesquisa exploratória buscou verificar como o componente de Revisão por Pares do Moodle funcionava, obtendo ideias para construir uma ferramenta de revisão por pares para o aprendizado de diagramas UML. As etapas de aplicação da técnica no Moodle foram as mesmas cumpridas na sala de aula. No entanto, apenas a turma de ERAS do quinto período participou. Os alunos deveriam submeter seus diagramas de casos de uso no Moodle e em seguida os alunos revisavam os diagramas da UML de seus colegas. Para esta etapa cada aluno deveria avaliar dois diagramas de outros dois colegas. E, por fim, adaptavam seus diagramas de acordo com as observações feitas pelos revisores. No total houve 13 submissões de diagramas. Com a aplicação da técnica foi possível observar algumas limitações existentes no componente atual que serviram de base para a elaboração do conjunto de requisitos apresentados neste trabalho.

As limitações do encontradas no Moodle foram: não retornar as anotações feitas pelos revisores aos pares; não poder fazer anotações no próprio diagrama; não poder colocar um checklist como peer review information; não poder colocar uma escala de "sim" e "não" e não se aplica para cada do peer review information; não poder enviar a versão corrigida como versão final; não permitir a anonimidade do autor do diagrama; não permitir a opção "não se aplica" para um item do peer review information, e; não permitir mais de uma rodada de revisão. As limitações do Moodle em relação a aplicação da técnica de Revisão por Pares foram fatores preponderantes para a elicitação de requisitos. Na seção a seguir é apresentado o processo de elicitação de requisitos e o conjunto mencionado.

\section{Processo de Elicitação de Requisitos de Ferramenta para Revisão por Pares}

Verificada a adequação da técnica de Revisão por Pares na aprendizagem de modelagem de sistemas e, a partir da análise do recurso "Laboratório de Avaliação" disponibilizado no Moodle, observou-se uma série de oportunidades para o desenvolvimento de uma ferramenta para suporte à técnica em aulas de modelagem de sistemas. Para tanto, fez-se necessária a aplicação de algumas técnicas de elicitação de requisitos que estão detalhadas a seguir.

\subsection{Brainstorm e Entrevista}

Após a observação da técnica por meio da pesquisa exploratória, verificando sua viabilidade de aplicação em sala de aula e também as limitações do plugin do Moodle foi 
VIII Congresso Brasileiro de Informática na Educação (CBIE 2019)

Anais do XXX Simpósio Brasileiro de Informática na Educação (SBIE 2019)

realizado com a turma de FES um Brainstorm e com a turma de ERAS uma entrevista em formato de Focus Group.

As técnicas de entrevista em grupo mais comuns são a modalidade focal, de brainstorm ou chuva de ideias, a nominal e a de projeção [Minayo and Costa 2018]. Por esse motivo, o brainstorm foi utilizado como técnica de elicitação de requisitos nesta pesquisa. No Brainstorm os pesquisadores perguntaram aos participantes quais os pontos positivos e negativos de terem utilizado a técnica. Após isso, foi perguntado que ideias eles teriam para construir um software para a técnica de aprendizagem colaborativa utilizada na disciplina. Os alunos foram bastante participativos, eles falavam o que acreditavam ser necessário e os itens eram anotados no quadro branco. Ao final, cada item foi discutido com a própria turma, verificando o que era ou não requisito para o sistema.

Já o Focus Group se constitui num tipo de entrevista com um pequeno número de pessoas. O termo focal assinala que se trata de um encontro para aprofundamento em algum tema (o foco), para o qual a lente do pesquisador está apontada [Minayo and Costa 2018]. Por se tratar de um número menor de alunos na turma de ERAS, optou-se por utilizar o Focus Group para elicitar os requisitos com eles. Após o consentimento, os alunos foram filmados por duas câmeras enquanto participavam da dinâmica. O quadro foi dividido em vantagens e desvantagens enquanto para cada aluno foi entregue um bloquinho de post-its. O moderador começou a dinâmica relembrando a técnica e perguntando o que haviam achado dela.

Em seguida, um tempo foi disponibilizado para que cada aluno anotasse as vantagens e desvantagens de ter utilizado a técnica. Ao terminar, os alunos liam em voz alta suas vantagens e desvantagens e colavam o post-it no quadro branco. Com isto, terminou a fase de elicitação dos requisitos para uma ferramenta de Revisão por Pares para aprendizagem colaborativa de diagramas UML. Na seção a seguir, são apresentados os resultados do processo de elicitação de requisitos, que remete ao conjunto de requisitos para a ferramenta de Revisão por Pares e uma prototipação concebida a partir do conjunto apresentado.

\section{Resultados}

O principal resultado desta pesquisa, contendo os requisitos funcionais, para um sistema que dê suporte a Revisão por Pares de diagramas UML foi reunido após a aplicação das técnicas de elicitação apresentadas. Com os requisitos já definidos é possível discutir para qual plataforma desenvolver a ferramenta e quais tecnologias utilizar durante o desenvolvimento. Pensando nisso, foi desenvolvido um protótipo de acordo com os requisitos elicitados. Para o mediador, é interessante a configuração de fase da ferramenta, para que o mesmo possa configurar as definições do laboratório como nome e descrição, e também cadastrar instruções para o envio como número de anexo e tamanho máximo de upload.

Os requisitos elicitados nesta pesquisa estão descritos na Tabela 2 que está organizada em três colunas a saber: a primeira representa o ID do requisito, a outra representa os requisitos e a terceira representa a origem do requisito. A coluna origem, da tabela, foi usada para mostrar qual ou quais os processos de elicitação resultou/resultaram no determinado requisito. Para facilitar a leitura da tabela foram utilizadas abreviaturas para representar as técnicas, conforme segue: PE - Pesquisa Exploratória, B - Brainstorm, E Entrevista e ASC - Análise de Sistemas Correlatos. 
VIII Congresso Brasileiro de Informática na Educação (CBIE 2019)

Anais do XXX Simpósio Brasileiro de Informática na Educação (SBIE 2019)

Tabela 2. Requisitos resultantes do processo de elicitação.

\begin{tabular}{|c|c|c|}
\hline ID & REQUISITOS & ORIGEM \\
\hline 1 & $\begin{array}{l}\text { Possibilitar ao mediador que possa distribuir os trabalhos aos } \\
\text { revisores }\end{array}$ & [ASC] \\
\hline 2 & Possibilitar ao mediador o cadastro de um peer review information & [PE] \\
\hline 3 & Possibilitar quantas rodadas de peer review sejam necessárias & {$[\mathrm{B}, \mathrm{E}]$} \\
\hline 4 & $\begin{array}{l}\text { Possibilitar ao mediador o cadastro ou não da anonimidade dos } \\
\text { autores e revisores durante o processo de revisão }\end{array}$ & $\begin{array}{l}{[\mathrm{PE}, \mathrm{B}} \\
\mathrm{E}, \mathrm{ASC}]\end{array}$ \\
\hline 5 & Possibilitar ao mediador o cadastro de texto base sobre a atividade & [ASC] \\
\hline 6 & $\begin{array}{l}\text { Classificar o tipo do diagrama (casos de uso, classes, sequência e } \\
\text { etc.) }\end{array}$ & {$[\mathrm{PE}, \mathrm{ASC}]$} \\
\hline 7 & $\begin{array}{l}\text { Possibilitar ao mediador o cadastro de instruções para o envio e } \\
\text { avaliação }\end{array}$ & {$[\mathrm{PE}, \mathrm{ASC}]$} \\
\hline 8 & $\begin{array}{l}\text { Possibilitar ao mediador o cadastro de configuração } \\
\text { de notas (para avaliação e aprovação) }\end{array}$ & {$[\mathrm{PE}, \mathrm{ASC}]$} \\
\hline 9 & Possibilitar que o autor realize upload do arquivo para a revisão & [B] \\
\hline 10 & Possibilitar ao autor a visualização do feedback dado pelo revisor & [B] \\
\hline 11 & Possibilitar que o autor possa valiar o revisor (por estrelas) & [B] \\
\hline 12 & Possibilitar que o sistema possa distribuir os trabalhos aos revisores & [ASC] \\
\hline 13 & $\begin{array}{l}\text { Possibilitar que o revisor possa realizar a revisão de mais de um } \\
\text { diagrama }\end{array}$ & {$[\mathrm{E}]$} \\
\hline 14 & $\begin{array}{l}\text { Possibilitar que o revisor possa realizar o upload de um arquivo } \\
\text { revisado }\end{array}$ & {$[\mathrm{B}, \mathrm{E}]$} \\
\hline 15 & $\begin{array}{l}\text { Possibilitar que o revisor possa escrever sim, não ou não se aplica } \\
\text { para cada item do peer review caso esteja usando os checklists }\end{array}$ & {$[\mathrm{E}, \mathrm{ASC}]$} \\
\hline 16 & Possibilitar comentário do revisor referente a cada item avaliado & [PE, E] \\
\hline 17 & $\begin{array}{l}\text { Possibilitar que o revisor envie questionamentos ao autor do } \\
\text { diagrama }\end{array}$ & {$[\mathrm{E}, \mathrm{ASC}]$} \\
\hline 18 & Cadastrar usuário (autor/revisor e mediador) & $\begin{array}{l}{[\mathrm{B}, \mathrm{E},} \\
\mathrm{ASC}]\end{array}$ \\
\hline 19 & Possibilitar um sistema de ranking (melhor resposta) & [B] \\
\hline 20 & Garantir a não-avaliação & {$[\mathrm{B}, \mathrm{ASC}]$} \\
\hline
\end{tabular}

Diante disso, apresenta-se na Figura 2 a tela de submissão do diagrama para Revisão por Pares. Esta tela possibilita que o aluno veja as definições da atividade bem como informações sobre o envio (submissão), instruções e exemplos. O aluno pode, ainda, realizar o upload de sua resposta à atividade, que consiste em um arquivo contendo a imagem do diagrama desenvolvido. Esta tela consiste na principal funcionalidade do ponto de vista do autor do diagrama. Após o upload do arquivo, o autor pode salvar o seu diagrama e submetê-lo para a revisão. No menu é possível selecionar a fase em que a Revisão por Pares se encontra. As fases previstas consistem em: submissão, revisão e feedback. Na fase "submissão", os alunos verificam a atividade a ser realizada e fazem a submissão de suas respostas. Na fase "revisão", os alunos tornam-se revisores dos trabalhos submetidos por seus colegas e realizam a avaliação destes. Por fim, na fase "feedback", os alunos verificam as avaliações de seus trabalhos, realizam as devidas correções e os submete 
VIII Congresso Brasileiro de Informática na Educação (CBIE 2019)

Anais do XXX Simpósio Brasileiro de Informática na Educação (SBIE 2019)

como versão final. Os usuários da ferramenta contam ainda com uma opção chamada "Information"que tem como objetivo dar suporte à quem tiver dúvidas no uso da mesma.
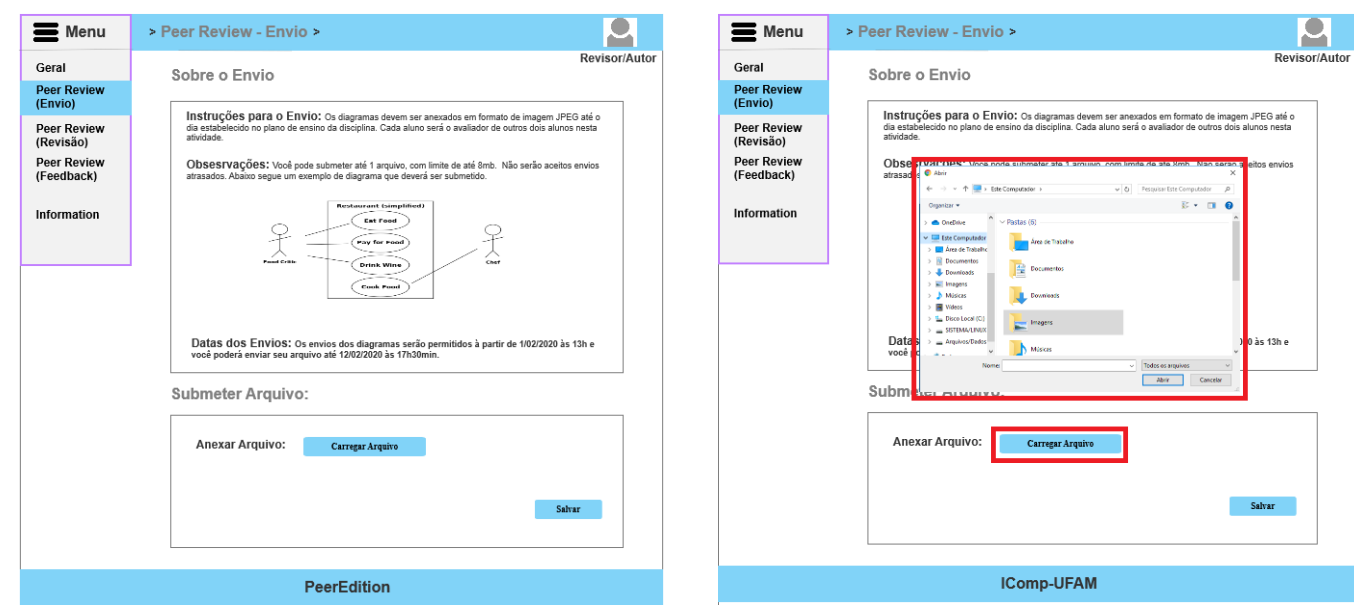

Figura 2. Tela onde o aluno deve responder a atividade para o professor

A Figura 3 mostra a tela do revisor. Nesta tela, o aluno torna-se revisor dos trabalhos dos colegas e pode visualizar as informações sobre o laboratório e sobre como deverá realizar a revisão dos trabalhos. Há, também, a opção de anexar um documento adicional sobre o diagrama revisado. No exemplo do protótipo o diagrama é anexado de forma análoga à submissão da tarefa conforme dito anteriormente. A ferramenta possibilita, ainda, a edição do diagrama e de comentários acerca da atividade revisada.
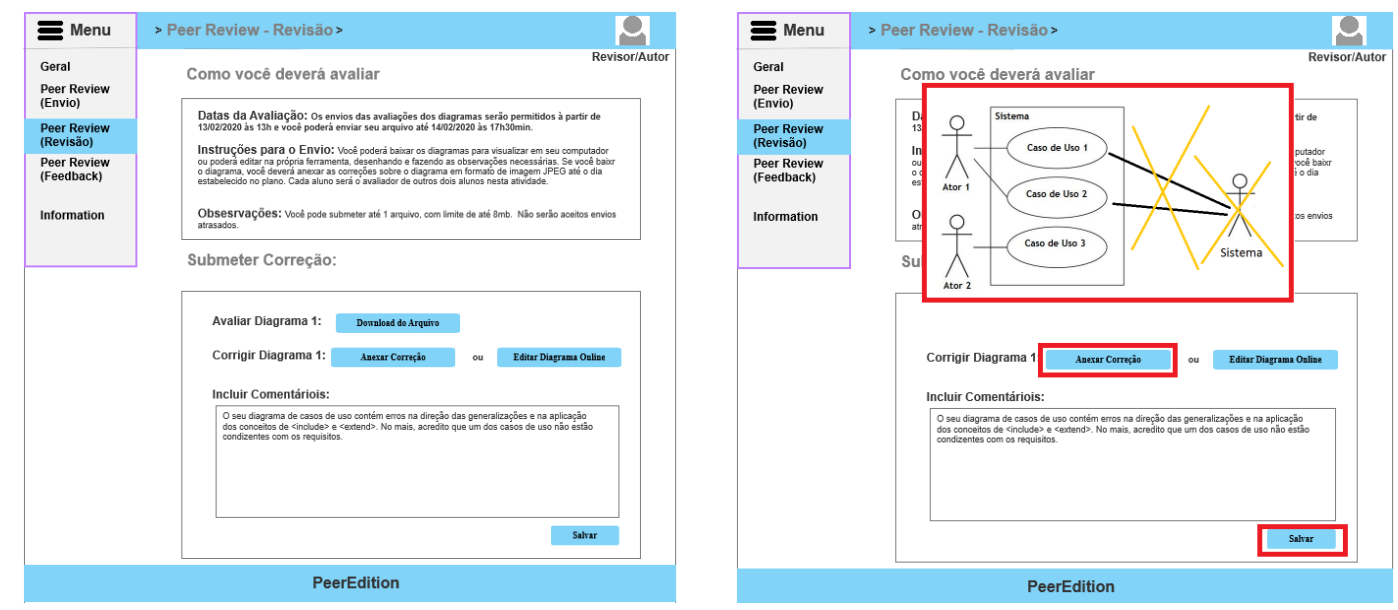

Figura 3. Tela onde o participante pode realizar a revisão do diagrama

Existem diversos trabalhos sobre Revisão por Pares, inclusive apresentando ferramentas, mas com este protótipo, foi possível verificar a visão geral do autor, do revisor e do mediador de uma revisão focando em modelagem de diagramas com UML. Além de permitir a coleta de dados básicos para implementação de uma ferramenta de Revisão por Pares, disseminando ideias de como distribuir as necessidades da técnica em uma ferramenta, sendo muito útil para validar os requisitos elicitados e saber como eles poderão ser dispostos no sistema. 
VIII Congresso Brasileiro de Informática na Educação (CBIE 2019)

Anais do XXX Simpósio Brasileiro de Informática na Educação (SBIE 2019)

\section{Conclusão}

Este trabalho teve como objetivo verificar a viabilidade da técnica de Revisão por Pares durante o aprendizado de modelagem de sistemas. Para isso, a técnica foi aplicada em três rodadas nas disciplinas do curso de Engenharia de Software e então os participantes responderam a um questionário sobre a sua experiência. Apesar de alguns alunos relatarem que preferem outros métodos de ensino, outros responderam que a técnica foi eficiente durante a aprendizagem e contribuiu para melhor compreensão do tema de estudo. Com a viabilidade da técnica, foi possível elicitar os requisitos para uma ferramenta que mediasse a Revisão por Pares, para ser utilizada durante o aprendizado de diagramas UML. As técnicas utilizadas com os alunos para elicitação foram Brainstorm e Entrevista. No entanto, utilizou-se também Pesquisa Exploratória e Análise de Sistemas Correlatos (considerando a aplicação da Revisão por Pares em uma das turmas utilizando o Moodle). Diante dos requisitos, foi possível conceber um protótipo para a ferramenta em questão. A etapa de prototipação teve como objetivo validar os requisitos existentes por meio de uma visão inicial da ferramenta. Os próximos passos desta pesquisa tratam da utilização em cenário real da ferramenta, verificando sua adequação considerando pontos de vista tanto dos alunos quanto dos professores de disciplinas de modelagem de software.

\section{Referências}

Barkley, E. F., Cross, K. P., and Major, C. H. (2014). Collaborative learning techniques: A handbook for college faculty. John Wiley \& Sons.

Bruning, E. (2016). Uma ferramenta de modelagem colaborativa de diagramas de classes.

Jenal, S., Willamowius Vituri, D., Machado Ezaías, G., Almeida da Silva, L., and Larcher Caliri, M. H. (2012). O processo de revisão por pares: uma revisão integrativa de literatura. Acta Paulista de Enfermagem, 25(5).

Junior, K., Sussumo, A., Roque, I., Prá, D., and Vinicius, W. J. (2016). Coavalieitor: sistema de suporte para avaliação por pares.

Ma, Z. (2017). An approach to improve the quality of object-oriented models from novice modelers through project practice. Frontiers of Computer Science, 11(3):485-498.

Minayo, M. C. d. S. and Costa, A. P. (2018). Fundamentos teóricos das técnicas de investigação qualitativa.

Neto, C., Sampaio, Á., et al. (2004). Análise de requisitos da revisão pelos pares na aprendizagem. Dissertação (Dissertação em Engenharia de Produção).

Quarto, C. C., Bercht, M., Nascimento, E., Veras, N., de Sousa Moraes, D., Busson, A., and Silva, F. (2017). Hermes: Um sistema de apoio à formação de grupos em ambientes de aprendizagem colaborativa. In Brazilian Symposium on Computers in Education (Simpósio Brasileiro de Informática na Educação-SBIE), volume 28, page 1697.

Silva, W., Gadelha, B., Steinmacher, I., and Conte, T. (2018). What are the differences between group and individual modeling when learning uml? In Proceedings of the XXXII Brazilian Symposium on Software Engineering, pages 308-317. ACM. 